\title{
Large graphene-induced shift of surface-plasmon resonances of gold films: Effective-medium theory for atomically thin materials
}

\author{
Md Kamrul Alam, ${ }^{1}$ Chao Niu, ${ }^{2}$ Yanan Wang,,${ }^{3,4}$ Wei Wang,,${ }^{5,6}$ Yang Li, ${ }^{4}$ Chong Dai, ${ }^{7}$ Tian Tong, ${ }^{4}$ Xiaonan Shan, ${ }^{4}$ \\ Earl Charlson, ${ }^{4}$ Steven Pei, ${ }^{4}$ Xiang-Tian Kong $\odot,{ }^{3}$ Yandi Hu, ${ }^{7}$ Alexey Belyanin, ${ }^{8}$ Gila Stein $\odot,{ }^{9}$ \\ Zhaoping Liu, ${ }^{5,6}$ Jonathan $\mathrm{Hu}^{2,}{ }^{2, *}$ Zhiming Wang, ${ }^{3,10}$ and Jiming Bao ${ }^{1,4, \dagger}$ \\ ${ }^{1}$ Materials Science and Engineering, University of Houston, Houston, Texas 77204, USA \\ ${ }^{2}$ Department of Electrical \& Computer Engineering, Baylor University, Waco, Texas 76798, USA \\ ${ }^{3}$ Institute of Fundamental and Frontier Sciences, University of Electronic Science and Technology of China, Chengdu, Sichuan 610054, China \\ ${ }^{4}$ Department of Electrical and Computer Engineering, University of Houston, Houston, Texas 77204, USA \\ ${ }^{5}$ Ningbo Institute of Materials Technology \& Engineering, Chinese Academy of Sciences, Ningbo, Zhejiang 315201, China \\ ${ }^{6}$ Key Laboratory of Graphene Technologies and Applications of Zhejiang Province, Ningbo Institute of Materials Technology and \\ Engineering, Chinese Academy of Sciences Ningbo, Zhejiang 315201, China \\ ${ }^{7}$ Department of Civil and Environmental Engineering, University of Houston, Houston, Texas 77204, USA \\ ${ }^{8}$ Department of Physics \& Astronomy, Texas A\&M University, College Station, Texas 77843, USA \\ ${ }^{9}$ Chemical and Biomolecular Engineering, The University of Tennessee, Knoxville, Tennessee 37996, USA \\ ${ }^{10}$ State Key Laboratory of Electronic Thin Films and Integrated Devices, University of Electronic Science and Technology of China, \\ Chengdu, Sichuan 610054, China
}

(Received 27 April 2019; revised manuscript received 28 July 2019; published 3 January 2020)

\begin{abstract}
Despite successful modeling of graphene as a 0.34-nm-thick optical film synthesized by exfoliation or chemical vapor deposition (CVD), graphene-induced shift of surface-plasmon resonance (SPR) of gold films has remained controversial. Here we report the resolution of this controversy by developing a clean CVD graphene transfer method and extending Maxwell-Garnett effective-medium theory (EMT) to two-dimensional (2D) materials. A SPR shift of $0.24^{\circ}$ is obtained and it agrees well with 2D EMT in which wrinkled graphene is treated as a 3-nm graphene/air layered composite, in agreement with the average roughness measured by atomic force microscopy. Because the anisotropic built-in boundary condition of 2D EMT is compatible with graphene's optical anisotropy, graphene can be modeled as a film thicker than $0.34 \mathrm{~nm}$ without changing its optical property; however, its actual roughness, i.e., effective thickness, will significantly alter its response to strong out-of-plane fields, leading to a larger SPR shift.
\end{abstract}

DOI: 10.1103/PhysRevResearch.2.013008

\section{INTRODUCTION}

Maxwell-Garnett effective-medium theory (EMT) was developed more than 100 years ago to obtain the macroscopic dielectric property of an inhomogeneous medium [1,2]. The Maxwell-Garnett (MG) mixing formula provides us the permittivity of a composite in terms of the permittivity and volume fraction of the individual constituents in a host medium [1-3]. The theory becomes more important today as nanostructures and nanomaterials are routinely synthesized and assembled to make nanocomposites or metamaterials for the desired electromagnetic responses and functionalities. Because the original mixing formula is based on noninteract-

\footnotetext{
*Jonathan_Hu@baylor.edu

${ }^{\dagger}$ Corresponding author: jbao@uh.edu
}

Published by the American Physical Society under the terms of the Creative Commons Attribution 4.0 International license. Further distribution of this work must maintain attribution to the author(s) and the published article's title, journal citation, and DOI. ing spherical inclusions in a host medium, it has been revised to handle nonspherical inclusions with mutual interaction [3-15]. The original and revised mixing formulas have been proven to be powerful tools in accurately capturing the macroscopic electromagnetic responses of composite materials, and good agreements have been demonstrated between theory and experiment for many systems such as metal-ceramic films [6,16], polymer-ceramic composites [17], amorphous silicon thin films [18], polymer-single-walled carbon nanotube composite [8], and aligned carbon nanotube film [19,20]. However, all these studies only investigated one- or threedimensional (3D) structures in three-dimensional host media; EMT for two-dimensional (2D) layered structures have not been evaluated thoroughly although the theory was developed long ago [21] and atomically thin 2D structures have become widely available.

Graphene, a truly atomically thin nanomaterial, has been treated as a 3D-like flat thin film with $n$ and $k$, real and imaginary parts of the refractive index, and with a finite thickness since its first optical characterization using spectroscopic ellipsometry [22]. Its picture as a $0.34-\mathrm{nm}$ film, no matter if it is exfoliated or grown by chemical vapor 
deposition (CVD), has worked very well in nearly all optical characterizations such as ellipsometry [22-27], attenuated total reflection (ATR) [28,29], and reflection spectroscopy [30,31]. Surface-plasmon resonance (SPR) of gold film in the Kretschmann configuration is sensitive to minute changes on a sample's surface, so it is an ideal tool to explore basic optoelectronic property of thin dielectric films and study their light-matter interactions. However, there has been a big discrepancy between theory and experiment. Based on the flat graphene picture, the SPR shift of an Au film in the resonant angle with and without single-layer graphene in air is calculated to be less than $0.1^{\circ}$ [32-38]. Experimentally, except for micrometer-size exfoliated graphene [39], the SPR shift induced by large-size CVD graphene is more than twice the calculated value, varying from $0.24^{\circ}$ to $1^{\circ}[15,40-42]$.

In this work, we apply an effective-medium theory to atomically thin material and report the resolution of the graphene SPR puzzle both experimentally and theoretically. We first develop a polymer-free CVD graphene transfer method to make sure that the SPR shift is induced by graphene only. We then point out several mistreatments in previous calculations, and good agreement is achieved using actual roughness of graphene and 2D EMT: Atomically thin materials should be treated as a flat film with effective thickness depending on its intrinsic surface roughness. Finally, we show that the Kretschmann configuration is an excellent platform to test 2D EMT and characterize anisotropic 2D composite films.

\section{RESULTS AND DISCUSSION}

Monolayer graphene was grown on polycrystalline $\mathrm{Cu}$ foils using a home-built CVD system [43]. In order to avoid any potential chemical contamination $[44,45]$, we developed a PMMA-free graphene transfer technique. Our approach took advantage of the hydrophobic nature of graphene, which makes graphene float on the etching solution without any polymer supporting layer [45]. Figure 1(a) shows a floating graphene/copper on aqueous iron nitrate etching solution. Red marks were placed on the corner of the graphene before etching to make it visible. After $\mathrm{Cu}$ was etched out, DI water was slowly added from the top, and the etching solution was drained from the bottom until it was completely removed [Figs. 1(b) and 1(c)]. One hundred milliliters of hydrochloric acid $(\mathrm{HCl} 5 \mathrm{M})$ was finally injected into the container and then replaced by DI water again to eliminate Fe residues. A floating graphene on DI water was scooped by a Au/glass substrate and could be transferred to any substrates in principle. This method is simpler than many reported polymer-free or support-free methods [46-51]. High-quality monolayer graphene was confirmed by Raman and optical transmission [52,53]; see the Supplemental Material [54].

Three types of $\mathrm{Au}$ films were first prepared and characterized with SPR before graphene transfer: regular Au film fabricated with electron-beam evaporation, regular film after thermal annealing, and template stripped gold (TSG) film [55]. They all had a nominal thickness of $45 \mathrm{~nm}$ and were evaporated (regular $\mathrm{Au}$ and annealed) or attached (TSG) to glass slides which are index matched with the SPR prism. Figure 1(d) shows the schematic of the Kretschmann configuration. A 633-nm HeNe laser was used to excite the surface
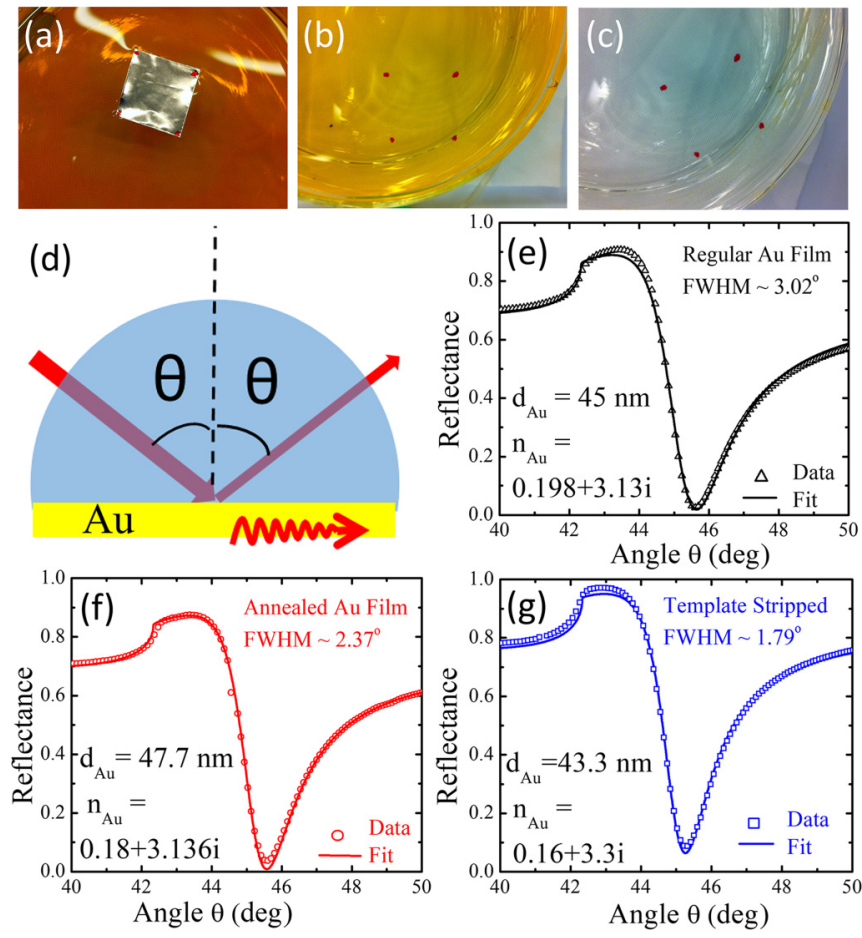

FIG. 1. PMMA-free graphene transfer and SPR of three Au films. (a) Graphene covered $\mathrm{Cu}$ foil floating on iron nitrate etching solution. (b), (c) Graphene floats on (b) etching solution and eventually on (c) DI water after gradual solution replacement. (d) Schematic of Kretschmann configuration with a hemisphere prism and Au film. (e)-(g) Surface-plasmon resonance (SPR) curves of three Au films and corresponding fitting curves and parameters. $n_{\text {glass }}=1.485 . n_{\mathrm{Au}}$ and $d_{\mathrm{Au}}$ are index and thickness of the Au film, respectively.

plasmon. Figures $1(\mathrm{e})-1(\mathrm{~g})$ show SPR curves of three $\mathrm{Au}$ films in ambient air. All of them display characteristic features with the minimum reflectance around $45.5^{\circ}$, indicating the excitation of SPR. These SPR curves can be well fitted by treating Au film as a homogeneous layer with adjustable index [56-59]. The obtained refractive indices (of the Au films are included in the figures, they are close to each other and fall within the typical index range of $\mathrm{Au}$ [59-61], indicating a successful modeling of Au film SPR.

A major advantage of SPR is that it is very sensitive to the dielectric environment of the Au film. Figures 2(a)-2(c) show SPR curves for the same three films after transferring monolayer graphene. For comparison, the initial curves without graphene are also included. It can be seen that graphene induced a significant change to each SPR curve with a similar SPR shift of $\sim 0.24^{\circ}$ despite different surface roughness of the three Au films. Similar measurements have been reported, but our shift is among the lowest with CVD graphene [15,40-42]. We believe this is due to our PMMA-free graphene transfer technique since any additional contamination will increase the SPR shift.

Graphene-induced SPR shift has also been calculated by many groups [32-38]. However, the calculated values are less than half of the lowest experimental value [15,40-42]. The predicted shift of $\sim 0.1^{\circ}$ is always reached when graphene is treated as a 0.34-nm-thick flat homogeneous film with a 

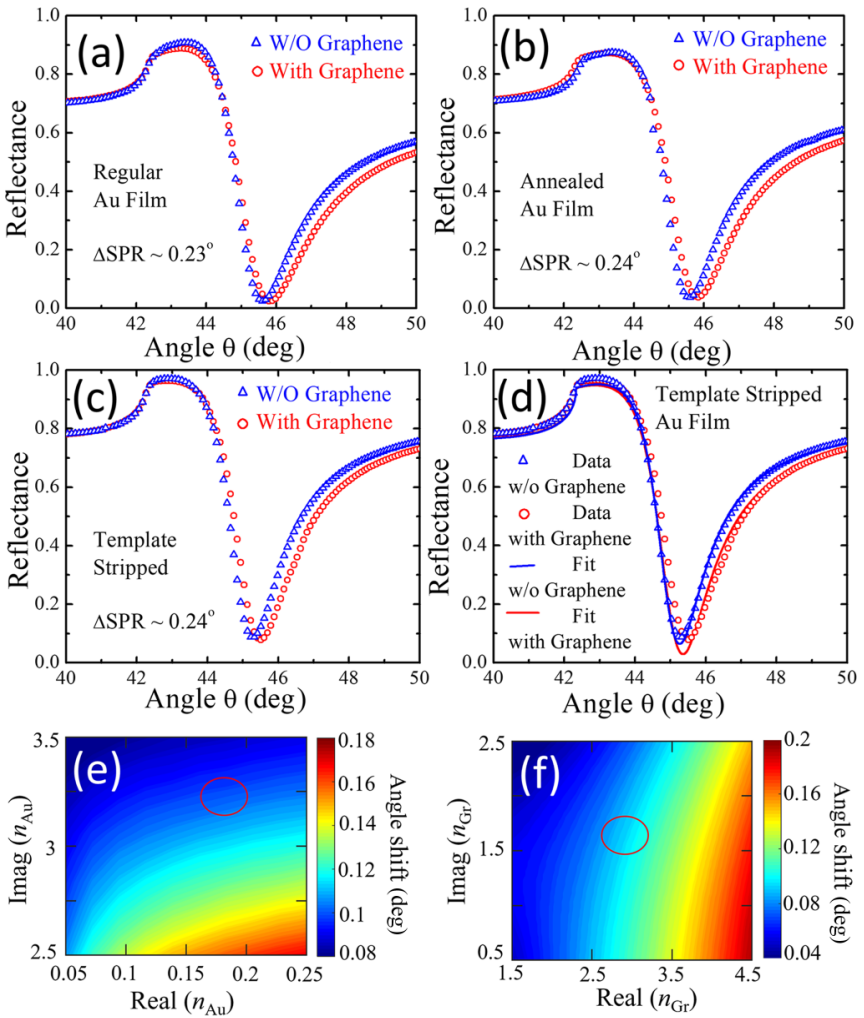

FIG. 2. Graphene-induced SPR shifts and simulations with variable $\mathrm{Au}$ and graphene index. (a)-(c) SPR shifts of the three Au films induced by monolayer graphene. (d) Measured and fitted SPR curves of TSG films (red) with and (blue) without graphene. Effect of refractive index of (e) Au and (f) graphene on the SPR shift. For (e), the index and thickness of graphene is fixed as $n_{\text {Graphene }}=$ $2.95+1.54 i$. For (f), the index and thickness of Au film is chosen to be $0.18+3.30 i$ and $45 \mathrm{~nm}$.

refractive index of $\sim 2.95+1.54 i$, which is an in-plane index obtained by ellipsometry [22,32-38]. The same SPR shift is obtained for our three types of Au films if we follow the same modeling approach. In fact, such a small SPR shift will always be obtained within experimental variation of index with Au films and graphene. To confirm previous calculations and prove the shortcomings of the flat graphene model, we calculate the SPR shift as functions of real and imaginary parts of the refractive indices of both graphene and Au film. Figures 2(e) and 2(f) show that the SPR shift varies smoothly and there is no hot spot with an abrupt large shift. For Au film, a change of $10 \%$ in the imaginary part has a larger effect than the change in the real part, but its imaginary part has very little variation among different Au films. For the typical index range of Au film and graphene (red circles on the images), SPR shift is found to be more or less around a small value of $0.10^{\circ}$.

This persistent disagreement on SPR shift between theory and experiment has existed for quite a while, but no serious attention has been paid to this issue. Obviously, the model of flat homogeneous graphene is oversimplified because graphene, especially grown by CVD, is not perfectly flat microscopically but tends to form wrinkles, nanoripples, and corrugations with surface roughness ranging from a few to $10 \mathrm{~nm}[62-71]$. As an atomically thin material, the flatness of graphene has been an interesting subject of research from its discovery. It was found both theoretically and experimentally that perfectly flat graphene cannot exist, long-wavelength thermal fluctuations destroy the long-range order of 2D crystals, and that graphene sheets exhibit intrinsic microscopic roughening where the surface normal varies by several degrees and out-of-plane deformations reach $1 \mathrm{~nm}[62,63]$. While this fundamental physical limit makes the initially perfect flat graphene obtained from mechanical exfoliation rough and uneven, the wrinkled structure in CVD graphene sheets reflects their actual morphological deformation that occurred during synthesis and transfer [64-71]. This is because in our CVD synthesis of a continuous film, the surfaces of copper foils are made of grains and are actually very rough microscopically. The surface of each grain is also not atomically flat because steps will develop at high temperature. Graphene tends to follow the morphology of the $\mathrm{Cu}$ surface; graphene films grown on $\mathrm{Cu}$ foils are inherently not flat. Even when copper is atomically flat initially, wrinkles will form on graphene film during the CVD cooling-down process because graphene has a much smaller thermal expansion coefficient than copper. Additional roughness will also be introduced during the graphene transfer process.

This can be seen from representative AFM images in Figs. 3(a) and 3(b). The graphene is placed on a TSG substrate, which has a surface roughness about $0.3 \mathrm{~nm}$ [55], so the observed roughness is an intrinsic property of our CVD graphene. Nevertheless, graphene is still well aligned in the same plane with an out-of-plane tilt angle less than $\sim 5^{\circ}$ for more than $90 \%$ of the total area. If we approximate graphene as a rippled sheet embedded in a thin layer of air, then we can use the MG mixing formula to calculate its effective $n$ and $k$ and then use them to calculate the SPR shift. The actual effective thickness $d$ of graphene/air can be estimated from the AFM image, and it determines the graphene volume fraction or filling factor. Because graphene is relatively flat, it is safe to assume that the total amount of graphene is the same as a monolayer. The volume fraction $f=t / d$, where $t=0.34 \mathrm{~nm}$ is the thickness of monolayer graphene and $d$ is the effective thickness. This simple form of filling factor is due to the nature of $2 \mathrm{D}$ inclusion and the contribution of single-layer graphene to the graphene/air composite. Thus, the dielectric constant of the composite will be given by [3]

$$
\varepsilon_{\mathrm{eff}}=\frac{1+2 f \frac{\varepsilon-1}{\varepsilon+2}}{1-f \frac{\varepsilon-1}{\varepsilon+2}},
$$

where $\varepsilon=(2.95+1.54 i)^{2}=6.33+9.09 i$. Figure 3(c) plots the thickness-dependent effective $n$ and $k$ of graphene/air based on Eq. (1). Both $n$ and $k$ decrease quickly as $d$ increases, while $k$ decreases much faster than $n$, dropping by more than half when the $d$ doubles. Figure 3(d) shows an example of a SPR curve when $d$ is $10.2 \mathrm{~nm}$. A SPR shift of $0.17^{\circ}$ is obtained. This shift is much larger than that with the flat graphene; however, when we calculate the thicknessdependent SPR shift as shown in Fig. 3(e), we found a maximum shift of $0.17^{\circ}$ regardless of the roughness of the graphene. As $d$ goes beyond $10 \mathrm{~nm}$, the SPR shift starts to 


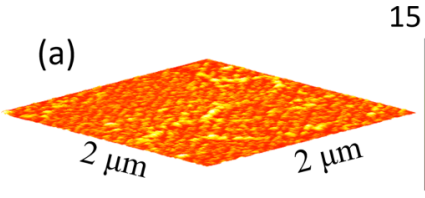

$15 \mathrm{~nm}$
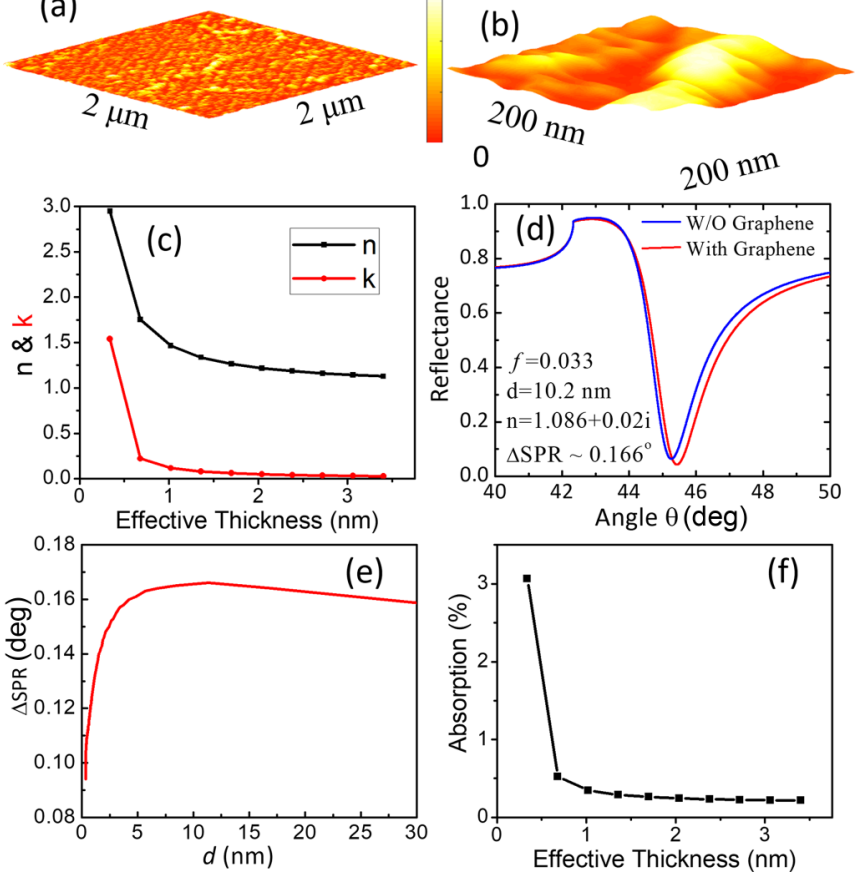

FIG. 3. Graphene AFM images and Maxwell-Garnett EMT. (a) 3D AFM image of graphene with a $2 \times 2 \mu \mathrm{m}^{2}$ scan area on the TSG Au substrate. Root mean square (RMS) roughness is 3.02 $\mathrm{nm}$. (b) Zoomed-in image of the roughest center region in (a). (c) $n$ and $k$ of graphene/air composite as a function of effective thickness $d$. (d) Representative example of SPR curves with and without graphene/air composite film with an effective thickness $d$ of $10.2 \mathrm{~nm}$. (e) Calculated SPR shift as a function of effective thickness $d$ of graphene/air composite. (f) Thickness-dependent optical absorbance of graphene/air composite calculated by $A=\frac{4 \pi n k d}{\lambda}$.

decrease, making it impossible to reach $0.24^{\circ}$. This failure of MG theory can also be seen from the sharp decrease in optical absorption of the graphene/air composite in Fig. 3(f). Experimentally, for all the graphene, no matter whether it is grown by exfoliation or CVD, the transmission is kept at $\sim 97 \%$ [72,73]. This failure of MG EMT is anticipated because graphene is not a spherical inclusion, and graphene's dielectric constant is intrinsically not isotropic [22].

Since graphene is nearly parallel to the Au film, we can approximate it as a graphene/air layered composite. Such layered or stratified composite was initially studied theoretically by Rytov in 1956 [21]. Unlike isotropic spherical inclusions, Figure. 4(a) shows the anisotropic boundary condition for $E$ and $D$, indicating that $2 \mathrm{D}$ layered composite is intrinsically anisotropic even if each constituent material is isotropic. It is important to point out that graphene is still quite flat [Fig. 4(b)]; it is also anisotropic, although it has been treated as an isotropic medium in previous calculations of SPR shift [32-38]. The effective dielectric constant can be conveniently derived based on its definition and the Maxwell equation's boundary conditions: $\varepsilon_{\text {eff }}$ is the ratio of average electric flux density $D$ to average electric field $E$; $E$ is continuous in the parallel direction) and $D$ is continuous in the perpendicular direction [3].
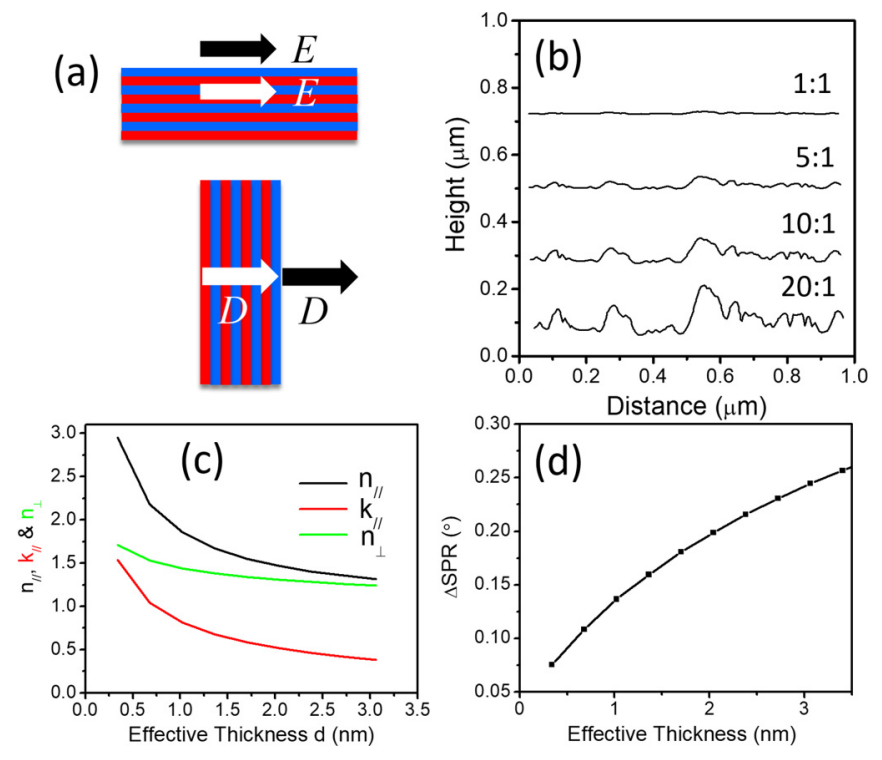

FIG. 4. Effective-medium theory for 2D layered composite and its application to graphene SPR shift. (a) Boundary condition. The tangential component of the electric field $E$ and the normal component of the displacement $D$ are continuous. (b) Line scan across the center roughest region of the AFM in Figs. 3(a) and 3(b) at different height-to-distance scale. (c) $n$ and $k$ of graphene/air composite as a function of effective thickness $d$. (d) Calculated SPR shifts as a function of effective thickness $d$.

In the parallel direction, for two layered materials with volume fractions of $f_{a}$ and $f_{b}$, the average $D_{\|}$is given by

$$
\begin{aligned}
D_{\|} & =f_{a} D_{\| a}+f_{b} D_{\| b}=f_{a} \varepsilon_{\| a} E_{\| a}+f_{b} \varepsilon_{\| b} E_{\| b} \\
& =\left(f_{a} \varepsilon_{\| a}+f_{b} \varepsilon_{\| b}\right) E_{\|}=\epsilon_{\|} E_{\|} .
\end{aligned}
$$

Since $E_{\| a}=E_{\| b}=E_{\|}$, we obtain

$$
\epsilon_{\|}=f_{a} \varepsilon_{\| a}+f_{b} \varepsilon_{\| b} .
$$

In the perpendicular direction, we have the average electrical field $E_{\perp}$, which is given by

$$
\begin{aligned}
E_{\perp} & =f_{a} E_{\perp a}+f_{b} E_{\perp b}=f_{a} \frac{D_{\perp a}}{\varepsilon_{\perp a}}+f_{b} \frac{D_{\perp b}}{\varepsilon_{\perp b}} \\
& =\left(\frac{f_{a}}{\varepsilon_{\perp a}}+\frac{f_{b}}{\varepsilon_{\perp b}}\right) D_{\perp}=\frac{D_{\perp}}{\varepsilon_{\perp}} .
\end{aligned}
$$

Since $D_{\perp a}=D_{\perp b}=D_{\perp}$, we obtain

$$
\frac{1}{\varepsilon_{\perp}}=\frac{f_{a}}{\varepsilon_{\perp a}}+\frac{f_{b}}{\varepsilon_{\perp b}} .
$$

The volume fraction of graphene is still $0.34 / d$. Figure 4(c) shows the effective index of $n$ and $k$ in both parallel and perpendicular directions as $d$ increases. Note that because the out-of-plane optical absorption of singlelayer graphene is zero [22], effective $k$ in the perpendicular direction is also zero. Figure 4(d) shows the effect of effective thickness $d$ on the SPR shift. Because the index decreases much slower than in the 3D EMT case, a larger shift is achieved. Based on the SPR shift, the effective thickness should be around $3 \mathrm{~nm}$, which agrees with the average roughness of $3.02 \mathrm{~nm}$ calculated from the AFM image. Here we 
approximate the average roughness as the effective thickness of the graphene/air composite.

The success of 2D EMT can also be verified by many farfield optical observations of graphene. Because the imaginary part of air's dielectric constant is zero, Eq. (2) can be written as

$$
\operatorname{Im}\left(\epsilon_{\|}\right)=f \operatorname{Im}\left(\varepsilon_{\| a}\right)=\frac{t}{d} \operatorname{Im}\left(\varepsilon_{\| a}\right),
$$

where Im stands for the imaginary part of the variable. Since $\varepsilon=\varepsilon_{R}+i \varepsilon_{I}=(n+i k)^{2}$, we have $\varepsilon_{I}=2 n k$. Thus Eq. (4) can be written as

$$
2 n_{\|} k_{\|}=\frac{t}{d} 2 n_{\| g} k_{\| g}, \quad \text { i.e., } \quad d n_{\|} k_{\|}=t n_{\| g} k_{\| g} .
$$

Equation (5) shows that $d n_{\|} k_{\|}$is a constant regardless of the effective thickness $d$. According to Beer's law, the intensity of light travel through a thin film goes as $I(x)=$ $I_{o} e^{-\frac{4 \pi n k x}{\lambda}}$, so $d n_{\|} k_{\|}$determines the optical absorption of light through the film with a thickness $d$. In other words, absorption for the normal incident light through graphene is a constant no matter whether the graphene is flat or corrugated. This conclusion has been verified by our optical transmission spectrum (Fig. S2 in the Supplemental Material [54]) and numerous other experimental observations [72,74-77].

It is not surprising that a thicker graphene/air composite film from 2D EMT can have the same optical absorption as that of an original thin flat graphene. We further argue that this treatment of rough graphene does not affect any of its optical properties in conventional thin film optical characterizations when it is surrounded by dielectric media. This can be understood as follows. In principle graphene is an atomic network of carbon atoms; it should be treated as an infinitely thin sheet. A finite thickness of $0.34 \mathrm{~nm}$ is only a convenient choice, it can be varied in ellipsometry as long as $n$ and $k$ are also adjusted accordingly to fit the data. In this sense, the original picture of a flat 0.34 -nm-thick graphene is already an approximation. When we further increase its thickness using 2D effective-medium theory, we have kept the $E$ and $D$ boundary conditions the same as before. For conventional thin film optical characterizations, as long as the effective thickness is much smaller than the wavelength of light, the results remain the same.

However, the above argument becomes invalid when graphene is placed on the surface of a plasmonic or metallic film due to the following two reasons. First, the electrical field near the surface, called the near field, does not remain constant as when graphene is surrounded by a dielectric medium; instead, it changes rapidly over a short distance above the surface. Second, the near field is dominated by the field normal to the surface or graphene. These unique differences can be seen in Fig. 5(a) when the surface plasmon is excited by the incident laser. The color indicates the normalized intensity of the electric field and the arrows indicate the direction of the electric field. As a result, the out-of-the-plane dielectric constant of graphene matters, and previous calculations based on in-plane dielectric constant are not accurate; the effective thickness is also important: A thicker effective layer increases graphene interaction with a normal near field, leading to a larger SPR shift. To demonstrate this point, we calculate SPR
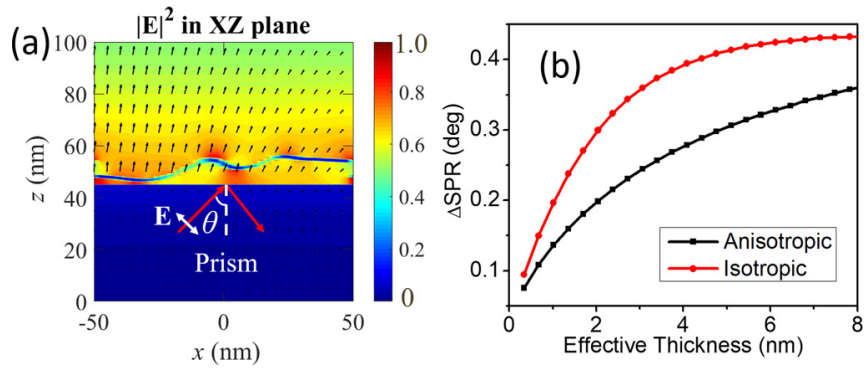

FIG. 5. The effect of perpendicular field and out-of-plane index on the SPR shift. (a) Cross-sectional view of electric field near the $\mathrm{Au}$ surface at a critical incident angle of $45.5^{\circ}$. The wrinkled line is graphene from AFM images in Figs. 3(a) and 3(b). (b) Thicknessdependent SPR shifts when graphene/air composite is treated as an isotropic or anisotropic media.

shifts as a function of the effective thickness in isotropic and anisotropic cases. Figure 5(b) shows that the difference increases as the thickness increases. For isotropic treatment, the in-plane optical constant from 2D EMT is used as both in-plane and out-plane constants. The isotropic calculation clearly overestimates the shift because the out-of-plane index is much smaller than the in-plane optical constant. Certainly, this treatment of isotropic $2 \mathrm{D}$ media is not self-consistent. Note that for a large effective thickness, 2D EMT also becomes invalid because the composite cannot be approximated as a layered structure defined in Fig. 4(a).

\section{CONCLUSIONS}

In summary, we have successfully extended traditional MG mixing theory for $3 \mathrm{D}$ isotropic media to $2 \mathrm{D}$ layered structures and applied 2D EMT to graphene. Good agreement of graphene-induced SPR shift between theory and experiment is achieved after wrinkled graphene is treated as an anisotropic graphene/air layered composite. We also revealed a unique property of 2D EMT: Normal incident optical absorption and typical optical properties remain the same regardless of the effective thickness of the layered composite. We point out that previous treatments of graphene as an isotropic medium are not accurate, and that the Kretschmann configuration is an excellent platform to measure the anisotropic optical constant of 2D material and test 2D effective-medium theory due to its strong normal near field on the surface. This picture of graphene as an effective medium is applicable to other atomically thin nanomaterials or layered structures such as graphene oxide (GO), reduced GO, transition metal dichalcogenides, and 2D material-based nanocomposite or metamaterials, and it helps to understand their electromagnetic responses and functionalities such as enhanced SPR sensitivity [33,34,78-84].

\section{ACKNOWLEDGMENTS}

J.M.B. acknowledges support from the Welch Foundation (Grant No. E-1728) and the National Science Foundation (Grant No. EEC-1530753). J.H. acknowledges support from the National Science Foundation (Grant No. ECCS-1809622).

M.K.A. and C.N. contributed equally to this work. 
[1] J. C. M. Garnett, Philos. Trans. R. Soc., A 203, 385 (1904).

[2] J. C. M. Garnett, Philos. Trans. R. Soc., A 205, 237 (1906).

[3] V. A. Markel, J. Opt. Soc. Am. A 33, 1244 (2016).

[4] W. Lamb, D. M. Wood, and N. W. Ashcroft, Phys. Rev. B 21, 2248 (1980).

[5] R. Ruppin, Opt. Commun. 182, 273 (2000).

[6] C. A. Foss, M. J. Tierney, and C. R. Martin, J. Phys. Chem 96, 9001 (1992).

[7] D. Schmidt and M. Schubert, J. Appl. Phys. 114, 083510 (2013).

[8] E. Kymakis and G. A. J. Amaratunga, Synth. Met. 142, 161 (2004).

[9] S. Giordano, J. Electrost. 58, 59 (2003).

[10] S. Riikonen, I. Romero, and F. J. Garcia de Abajo, Phys. Rev. B 71, 235104 (2005).

[11] V. Yannopapas and A. Moroz, J. Phys.: Condens. Matter 17, 3717 (2005).

[12] Y. Wang, G. W. Duan, L. Y. Zhang, L. H. Ma, X. G. Zhao, and X. Zhang, Sci. Rep. 8, 2087 (2018).

[13] I. Tsukerman, Phys. Lett. A 381, 1635 (2017).

[14] B. M. Wells, W. Guo, and V. A. Podolskiy, MRS Commun. 6, 23 (2016).

[15] O. Salihoglu, S. Balci, and C. Kocabas, Appl. Phys. Lett. 100, 213110 (2012).

[16] G. A. Niklasson, C. G. Granqvist, and O. Hunderi, Appl. Opt. 20, 26 (1981).

[17] Y. Rao, J. M. Qu, T. Marinis, and C. P. Wong, IEEE Trans. Compon. Packag. Technol. 23, 434 (2000).

[18] H. Fujiwara, J. Koh, P. I. Rovira, and R. W. Collins, Phys. Rev. B 61, 10832 (2000).

[19] F. J. Garcia-Vidal, J. M. Pitarke, and J. B. Pendry, Phys. Rev. Lett. 78, 4289 (1997)

[20] W. A. Deheer, W. S. Bacsa, A. Chatelain, T. Gerfin, R. Humphreybaker, L. Forro, and D. Ugarte, Science 268, 845 (1995).

[21] S. M. Rytov, Zh. Eksp. Teor. Fiz. 29, 605 (1956) [Sov. Phys. JETP 2, 466 (1956)].

[22] V. G. Kravets, A. N. Grigorenko, R. R. Nair, P. Blake, S. Anissimova, K. S. Novoselov, and A. K. Geim, Phys. Rev. B 81, 155413 (2010).

[23] G. E. Jellison, J. D. Hunn, and H. N. Lee, Phys. Rev. B 76, 085125 (2007).

[24] A. Matković, A. Beltaos, M. Milićević, U. Ralević, B. Vasić, D. Jovanović, and R. Gajić, J. Appl. Phys. 112, 123523 (2012).

[25] F. J. Nelson, V. K. Kamineni, T. Zhang, E. S. Comfort, J. U. Lee, and A. C. Diebold, Appl. Phys. Lett. 97, 253110 (2010).

[26] J. W. Weber, V. E. Calado, and M. C. M. van de Sanden, Appl. Phys. Lett. 97, 091904 (2010).

[27] U. Wurstbauer, C. Röling, U. Wurstbauer, W. Wegscheider, M. Vaupel, P. H. Thiesen, and D. Weiss, Appl. Phys. Lett. 97, 231901 (2010).

[28] S. Cheon, K. D. Kihm, H. g. Kim, G. Lim, J. S. Park, and J. S. Lee, Sci. Rep. 4, 6364 (2014).

[29] Q. Ye, J. Wang, Z. Liu, Z.-C. Deng, X.-T. Kong, F. Xing, X.-D. Chen, W.-Y. Zhou, C.-P. Zhang, and J.-G. Tian, Appl. Phys. Lett. 102, 021912 (2013).

[30] Z. H. Ni, H. M. Wang, J. Kasim, H. M. Fan, T. Yu, Y. H. Wu, Y. P. Feng, and Z. X. Shen, Nano Lett. 7, 2758 (2007).

[31] M. Bruna and S. Borini, Appl. Phys. Lett. 94, 031901 (2009).
[32] L. Wu, H. S. Chu, W. S. Koh, and E. P. Li, Opt. Express 18, 14395 (2010).

[33] S. Szunerits, N. Maalouli, E. Wijaya, J.-P. Vilcot, and R. Boukherroub, Anal. Bioanal. Chem. 405, 1435 (2013).

[34] P. K. Maharana, P. Padhy, and R. Jha, IEEE Photonics Technol. Lett. 25, 2156 (2013).

[35] S. H. Choi, Y. L. Kim, and K. M. Byun, Opt. Express 19, 458 (2011).

[36] A. Verma, A. Prakash, and R. Tripathi, Opt. Quantum Electron. 47, 1197 (2015).

[37] J. B. Maurya, Y. K. Prajapati, V. Singh, and J. P. Saini, Appl. Phys. A: Mater. Sci. Process. 121, 525 (2015).

[38] G. Mohanty, B. K. Sahoo, and J. Akhtar, Opt. Quantum Electron. 47, 1911 (2015).

[39] X. N. Shan, S. Chen, H. Wang, Z. X. Chen, Y. Guan, Y. X. Wang, S. P. Wang, H. Y. Chen, and N. J. Tao, Adv. Mater. 27, 6213 (2015).

[40] Y.-M. Bahk, G. Ramakrishnan, J. Choi, H. Song, G. Choi, Y. H. Kim, K. J. Ahn, D.-S. Kim, and P. C. M. Planken, ACS Nano 8, 9089 (2014).

[41] H. Jussila, H. Yang, N. Granqvist, and Z. Sun, Optica 3, 151 (2016).

[42] K. Chung, J. S. Lee, E. Kim, K. E. Lee, K. Kim, J. Lee, D. Kim, S. O. Kim, S. Jeon, H. Park, D. W. Kim, and D. H. Kim, Adv. Mater. Interfaces 5, 1800433 (2018).

[43] H. L. Cao, Q. K. Yu, L. A. Jauregui, J. Tian, W. Wu, Z. Liu, R. Jalilian, D. K. Benjamin, Z. Jiang, J. Bao, S. S. Pei, and Y. P. Chen, Appl. Phys. Lett. 96, 122106 (2010).

[44] Z. H. Liu, X. X. Lu, P. Peng, W. Wu, S. S. Pei, Q. K. Yu, and J. M. Bao, Phys. Rev. B 82, 155435 (2010).

[45] X. Li, Y. Zhu, W. Cai, M. Borysiak, B. Han, D. Chen, R. D. Piner, L. Colombo, and R. S. Ruoff, Nano Lett. 9, 4359 (2009).

[46] X.-D. Chen, Z.-B. Liu, C.-Y. Zheng, F. Xing, X.-Q. Yan, Y. Chen, and J.-G. Tian, Carbon 56, 271 (2013).

[47] P. Gupta, P. D. Dongare, S. Grover, S. Dubey, H. Mamgain, A. Bhattacharya, and M. M. Deshmukh, Sci. Rep. 4, 3882 (2014).

[48] X. Liang, B. A. Sperling, I. Calizo, G. Cheng, C. A. Hacker, Q. Zhang, Y. Obeng, K. Yan, H. Peng, Q. Li, X. Zhu, H. Yuan, A. R. Hight Walker, Z. Liu, L.-m. Peng, and C. A. Richter, ACS Nano 5, 9144 (2011).

[49] W. Regan, N. Alem, B. Alemán, B. Geng, Ç. Girit, L. Maserati, F. Wang, M. Crommie, and A. Zettl, Appl. Phys. Lett. 96, 113102 (2010).

[50] G. Zhang, A. G. Güell, P. M. Kirkman, R. A. Lazenby, T. S. Miller, and P. R. Unwin, ACS Appl. Mater. Interfaces 8, 8008 (2016).

[51] D. Y. Wang, I. S. Huang, P. H. Ho, S. S. Li, Y. C. Yeh, D. W. Wang, W. L. Chen, Y. Y. Lee, Y. M. Chang, C. C. Chen, C. T. Liang, and C. W. Chen, Adv. Mater. 25, 4521 (2013).

[52] Y. N. Wang, Z. H. Su, W. Wu, S. Nie, N. Xie, H. Q. Gong, Y. Guo, J. H. Lee, S. R. Xing, X. X. Lu, H. Y. Wang, X. H. Lu, K. McCarty, S. S. Pei, F. Robles-Hernandez, V. G. Hadjiev, and J. M. Bao, Appl. Phys. Lett. 103, 123101 (2013).

[53] Q. K. Yu, L. A. Jauregui, W. Wu, R. Colby, J. F. Tian, Z. H. Su, H. L. Cao, Z. H. Liu, D. Pandey, D. G. Wei, T. F. Chung, P. Peng, N. P. Guisinger, E. A. Stach, J. M. Bao, S. S. Pei, and Y. P. Chen, Nat. Mater. 10, 443 (2011).

[54] See Supplemental Material at http://link.aps.org/supplemental/ 10.1103/PhysRevResearch.2.013008 for Raman and optical transmission. 
[55] P. Nagpal, N. C. Lindquist, S. H. Oh, and D. J. Norris, Science 325, 594 (2009).

[56] T. Velinov, L. Ahtapodov, A. Nelson, M. Gateshki, and M. Bivolarska, Thin Solid Films 519, 2093 (2011).

[57] S. Zhang, L. Berguiga, J. Elezgaray, T. Roland, C. FaivreMoskalenko, and F. Argoul, Surf. Sci. 601, 5445 (2007).

[58] H. Liu, B. Wang, E. S. P. Leong, P. Yang, Y. Zong, G. Si, J. Teng, and S. A. Maier, ACS Nano 4, 3139 (2010).

[59] A. Rueda, N. Vogel, and M. Kreiter, Surf. Sci. 603, 491 (2009).

[60] P. B. Johnson and R. W. Christy, Phys. Rev. B 6, 4370 (1972).

[61] R. L. Olmon, B. Slovick, T. W. Johnson, D. Shelton, S.-H. Oh, G. D. Boreman, and M. B. Raschke, Phys. Rev. B 86, 235147 (2012).

[62] J. C. Meyer, A. K. Geim, M. I. Katsnelson, K. S. Novoselov, T. J. Booth, and S. Roth, Nature 446, 60 (2007).

[63] A. Fasolino, J. H. Los, and M. I. Katsnelson, Nat. Mater. 6, 858 (2007).

[64] L. Gao, W. Ren, H. Xu, L. Jin, Z. Wang, T. Ma, L.-P. Ma, Z. Zhang, Q. Fu, L.-M. Peng, X. Bao, and H.-M. Cheng, Nat. Commun. 3, 699 (2012).

[65] J. Kraus, S. Böcklein, R. Reichelt, S. Günther, B. Santos, T. O. Menteș, and A. Locatelli, Carbon 64, 377 (2013).

[66] N. Liu, Z. Pan, L. Fu, C. Zhang, B. Dai, and Z. Liu, Nano Res. 4, 996 (2011).

[67] G.-X. Ni, Y. Zheng, S. Bae, H. R. Kim, A. Pachoud, Y. S. Kim, C.-L. Tan, D. Im, J.-H. Ahn, B. H. Hong, and B. Özyilmaz, ACS Nano 6, 1158 (2012)

[68] Y. Zhang, T. Gao, Y. Gao, S. Xie, Q. Ji, K. Yan, H. Peng, and Z. Liu, ACS Nano 5, 4014 (2011).

[69] W. Zhu, T. Low, V. Perebeinos, A. A. Bol, Y. Zhu, H. Yan, J. Tersoff, and P. Avouris, Nano Lett. 12, 3431 (2012).

[70] V. E. Calado, G. F. Schneider, A. M. M. G. Theulings, C. Dekker, and L. M. K. Vandersypen, Appl. Phys. Lett. 101, 103116 (2012).
[71] L. Meng, Y. Su, D. Geng, G. Yu, Y. Liu, R.-F. Dou, J.-C. Nie, and L. He, Appl. Phys. Lett. 103, 251610 (2013).

[72] R. R. Nair, P. Blake, A. N. Grigorenko, K. S. Novoselov, T. J. Booth, T. Stauber, N. M. R. Peres, and A. K. Geim, Science 320, 1308 (2008).

[73] A. B. Kuzmenko, E. van Heumen, F. Carbone, and D. van der Marel, Phys. Rev. Lett. 100, 117401 (2008).

[74] K. S. Kim, Y. Zhao, H. Jang, S. Y. Lee, J. M. Kim, K. S. Kim, J.-H. Ahn, P. Kim, J.-Y. Choi, and B. H. Hong, Nature 457, 706 (2009).

[75] S. Bae, H. Kim, Y. Lee, X. Xu, J.-S. Park, Y. Zheng, J. Balakrishnan, T. Lei, H. Ri Kim, Y. I. Song, Y.-J. Kim, K. S. Kim, B. Özyilmaz, J.-H. Ahn, B. H. Hong, and S. Iijima, Nat. Nanotechnol. 5, 574 (2010).

[76] F. Bonaccorso, Z. Sun, T. Hasan, and A. C. Ferrari, Nat Photonics 4, 611 (2010)

[77] Z. Q. Luo, J. Z. Shang, S. H. Lim, D. H. Li, Q. H. Xiong, Z. X. Shen, J. Y. Lin, and T. Yu, Appl. Phys. Lett. 97, 233111 (2010).

[78] Y. Ryu, S. Moon, Y. Oh, Y. Kim, T. Lee, D. H. Kim, and D. Kim, Appl. Opt. 53, 1419 (2014).

[79] N. F. Lokman, A. A. A. Bakar, F. Suja, H. Abdullah, W. B. W. A. Rahman, N.-M. Huang, and M. H. Yaacob, Sens. Actuators, B 195, 459 (2014).

[80] X. Luo, T. Qiu, W. Lu, and Z. Ni, Mater. Sci. Eng., R 74, 351 (2013).

[81] M. Singh, M. Holzinger, M. Tabrizian, S. Winters, N. C. Berner, S. Cosnier, and G. S. Duesberg, J. Am. Chem. Soc. 137, 2800 (2015).

[82] S. Zeng, K. V. Sreekanth, J. Shang, T. Yu, C.-K. Chen, F. Yin, D. Baillargeat, P. Coquet, H.-P. Ho, A. V. Kabashin, and K.-T. Yong, Adv. Mater. 27, 6163 (2015).

[83] E. Wijaya, N. Maalouli, R. Boukherroub, S. Szunerits, and J. P. Vilcot, Proc. SPIE 8424, 84240R (2012).

[84] A. Kuzyk, R. Schreiber, H. Zhang, A. O. Govorov, T. Liedl, and N. Liu, Nat. Mater. 13, 862 (2014). 